\section{BfDI Peter Schaar: Die Europäische Datenschutzreform muss zügig abgeschlossen werden}

Der Bundesbeauftragte für den Datenschutz und die Informationsfreiheit Peter Schaar hält die Vorschläge des Innenausschusses des Europäischen Parlaments ${ }^{1}$ vom 21.10.2013 für ein wichtiges Signal. Die Reform des europäischen Datenschutzrechts wird damit einen entscheidenden Schritt vorangebracht.

Schaar: „Ich hoffe, dass die im Rat vertretenen Regierungen der 28 EU-Mitgliedstaaten dies als Chance begreifen, die Datenschutzreform zügig zu beschließen. Die Bundeskanzlerin sollte das Gelingen der Reform zur Chefsache machen!"

Die Vorschläge des Innenausschusses des Europäischen Parlaments enthalten nach Ansicht des Bundesdatenschutzbeauftragten eine Reihe von positiven Elementen, die sich vor allem von den inzwischen im Rat diskutierten Vorschlägen abheben:

- Datenübermittlungen an ausländische Behörden und Gerichte sollen nur noch auf der Basis von Rechtshilfeabkommen oder internationalen Vereinbarungen bei voller Transparenz gegenüber den Datenschutzbehörden erlaubt sein. Damit würde der Datenschutz beispielsweise gegenüber ausländischen Geheimdiensten deutlich gestärkt.

- Die Einwilligung soll als Ausdruck des Rechts auf informationelle Selbstbestimmung weiterhin eine zentrale Rolle bei der Verarbeitung personenbezogener Daten spielen. Anders als im Rat diskutiert, soll nur eine ausdrücklich erteilte Einwilligung wirksam sein. Der Zugang zu Dienstleistungen oder die Durchführung von Verträgen sollen nicht davon abhängig gemacht werden dürfen, dass die Betroffenen in eine Datenverarbeitung zu ganz anderen Zwecken einwilligen.

- Die Betroffenen sollen ihre Rechte leichter wahrnehmen können, indem sie auf einfache und standardisierte Art über die Verarbeitung ihrer personenbezogenen Daten informiert werden.

- Jeder soll grundsätzlich das Recht bekommen, einer Profilbildung, bei der seine Persönlichkeit durch automatisierte Datenverarbeitung bewertet wird, widersprechen zu können.

- Guter Datenschutz muss auch durch wirksame Sanktionen unterstützt werden. Der Innenausschuss schlägt ein differenziertes Sanktionssystem vor, mit dem auf unbedeutende, geringfügige Verstöße ebenso angemessen reagiert werden kann, wie auf schwerwiegende Verstöße, bei denen eine Geldbuße von bis zu fünf Prozent des jährlichen Umsatzes vorgesehen werden kann.

- Der Innenausschuss hält an der verpflichtenden Bestellung behördlicher und betrieblicher Datenschutzbeauftragter fest; eine Praxis, mit der Deutschland sehr gute Erfahrungen gemacht hat. Alle Unternehmen, deren Datenverarbeitung besondere Risiken hervorruft, sollen einen Datenschutzbeauftragten bestellen müssen.

1 http://www.janalbrecht.eu/fileadmin/material/Dokumente/DPR-Regulation-inofficial-consolidated-LIBE.pdf
Der Innenausschuss des Europäischen Parlaments hatte in seiner gestrigen Sitzung über das Mandat des zuständigen Berichterstatters für die Datenschutz-Grundverordnung zur Aufnahme von Verhandlungen mit dem Rat der Europäischen Union abgestimmt.

Die Änderungsvorschläge beziehen sich auf ein von der Europäischen Kommission im Januar 2012 vorgelegten Entwurf (KOM(2012) 11 endgültig), der eine aus dem Jahr 1995 stammende europäische Datenschutzrichtlinie 95/46/EG ablösen soll.

\section{Jan Philipp Albrecht ${ }^{2}$ zu den Ergebnissen der LIBE Ausschusssitzung}

„Ich freue mich über eine sehr breite Mehrheit für einen starken einheitlichen Datenschutz in der EU. Mit diesem Mandat kann das Europäische Parlament mit den Mitgliedsstaaten über die Einzelheiten einer EU-Datenschutzgrundverordnung verhandeln.

Nun liegt der Ball bei den Mitgliedsstaaten, die sich nach eineinhalb Jahren Debatte zügig auf eine Position einigen sollten. Bundeskanzlerin Angela Merkel hat die Verantwortung, beim EU-Gipfel in Brüssel Ende dieser Woche ihren Worten aus dem Wahlkampf Taten folgen zu lassen und die Verabschiedung der EU-Datenschutzgrundverordnung zum verbindlichen Ziel der EU-Staaten zu machen. Bislang gehörte die Bundesregierung zu den Bremsern der Datenschutzreform und hat es versäumt, auf ein hohes Niveau in der Verordnung zu drängen. Das starke Verhandlungsmandat des Europäischen Parlaments sollte Grund genug sein, dies jetzt nachzuholen.

Der Kompromiss ist ein großer Schritt für einen starken EU-Datenschutz: Künftig soll ausschließlich das neue EU-Recht gelten, wenn die Daten von Bürgerinnen und Bürgern in der EU verarbeitet werden, unabhängig davon, ob das Daten verarbeitende Unternehmen seinen Sitz in Deutschland, Irland, den USA oder anderswo hat. Außerdem müssen Google und Co Nutzerinnen und Nutzer öfter und explizit fragen, wenn sie deren Daten sammeln oder weitergeben wollen.

Wer möchte, dass eigene Daten gelöscht werden, soll ein „Recht auf Löschung" haben und dies auch durchsetzen können, solange sie nicht durch die Meinungs- und Informationsfreiheit geschützt sind. Ohne Rechtsgrundlage im EU-Recht dürfen Daten nicht mehr an Behörden in Drittstaaten weitergegeben werden. Unternehmen, die es dennoch tun - weil zum Beispiel die NSA sie dazu drängt - müssen mit scharfen Sanktionen rechnen. Die Datenschutzbeauftragten sollen zukünftig Strafen bis zu fünf Prozent des Jahresumsatzes eines Unternehmens verhängen dürfen.

Die Bundesregierung muss jetzt ihre Chance nutzen und Vorreiter für ein einheitliches hohes Datenschutzniveau werden. Nur dann wird es gelingen, den Bürgerinnen und Bürgern der EU noch

2 Jan Philipp Albrecht ist Abgeordneter des Europäischen Parlaments, Mitglied im Innenausschuss, stellvertretendes Mitglied im Rechtsausschuss und Verhandlungsführer des Europäischen Parlaments für die geplante Datenschutzverordnung. 